\title{
Enfoques de la gestión escolar: una aproximación desde el contexto latinoamericano
}

\section{Abel Pérez-Ruiz}

Universidad Pedagógica Nacional,

México

abe28ruiz@gmail.com

\section{Resumen}

El presente artículo hace un balance de los diferentes tratamientos y enfoques que se han desarrollado alrededor de la gestión escolar en los últimos años. El interés reside en identificar las distintas vertientes de análisis acerca de la nueva gestión pública aplicada a la educación como parte de los procesos de reforma educativa implementados recientemente en algunos países de América Latina. Sobre esa base, la idea de reorganizar a las escuelas se inserta dentro de un contexto de cambio que promueve la descentralización junto con mecanismos más flexibles para el funcionamiento de lo educativo. La presencia de estos principios ha dado lugar a resultados diversos y contrastantes que dan cuenta de realidades escolares heterogéneas, aun cuando las politicas públicas pretendan uniformarlas a partir de determinadas prescripciones normativas. Esta circunstancia hace que la gestión escolar sea todavía un tema inacabado y necesario de ahondar a fin de enriquecer su estudio correspondiente.

\section{Palabras clave}

Gestión educacional, educación, tendencia educacional. (Fuente: Tesauro de la Unesco).

Recepción: 2013-10-22 / Envío a pares: 2014-06-19 / Aceptación por pares: 2014-07-25 / Aprobación: 2014-08-17 DOI: $10.5294 /$ edu.2014.17.2.9

Para citar este artículo / To reference this article / Para citar este artigo

Pérez-Ruiz, A. (2014). Enfoques de la gestión escolar: una aproximación desde el contexto latinoamericano. Educ. Educ. 17 (2), $357-$ 369. Doi. 10.5294/edu.2014.17.2.9 


\title{
Approaches to School Management: An Approximation from the Latin American Perspective
}

\begin{abstract}
The article takes stock of the various tactics and approaches to school management developed in recent years. The objective is to identify the different approaches to analyzing the new form of public administration being applied to education, as part of the educational reform processes implemented recently in several Latin American countries. On that basis, the idea of reorganizing schools is embedded in a context of change that promotes decentralization, along with more flexible mechanisms for the way education operates. The existence of these principles has led to diverse and contrasting results that account for heterogeneous school realities, despite the existence of public policies that seek to align them according to certain regulatory standards or requirements. This means school management is still an unfinished topic and one requiring more in-depth consideration to enrich its study.
\end{abstract}

Key words

Educational management, educational trends, education. (Source: Unesco Thesaurus). 


\section{Enfoques da gestão escolar: uma aproximação a partir do contexto latino-americano}

Resumo

O presente artigo faz um balanço dos diferentes tratamentos e enfoques que têm sido desenvolvidos ao redor da gestão escolar nos últimos anos. O interesse reside em identificar as diferentes vertentes de análise sobre a nova gestão pública aplicada à educação como parte dos processos de reforma educativa implantados recentemente em alguns países da América Latina. Sobre essa base, a ideia de reorganizar as escolas insere-se num contexto de mudança que promove a descentralização junto com mecanismos mais flexiveis para o funcionamento do educativo. A presença desses princípios vem dando lugar a resultados diversos e contrastantes que dão conta de realidades escolares heterogêneas, mesmo que as politicas públicas pretendam uniformá-las a partir de determinadas prescrições normativas. Essa circunstância faz com que a gestão escolar seja ainda um tema inacabado e necessário aprofundar a fim de enriquecer seu estudo correspondente.

\section{Palavras-chave}

Gestão escolar, tendência educacional, educação. (Fonte: Tesauro da Unesco). 


\section{Introducción}

La gestión en los centros escolares, en tanto componente de una reordenación institucional, ha tenido como referencia un conjunto de cambios importantes en distintos órdenes de la vida social a lo largo de estos últimos años. Sin tratar de simplificar la complejidad y variedad de estas transformaciones en los diferentes países de América Latina, podemos situar cinco factores a nivel macro que han afectado el sentido de la educación en nuestros días. El primer factor lo representa — desde las décadas de los ochenta y noventa - la revisión de los fundamentos político-administrativos del Estado benefactor, lo cual ha dado pie a la promoción de diversas reformas estructurales centradas en el uso racional de los recursos y en la descentralización del aparato estatal. El segundo lo constituye el desplazamiento de una economía centrada en el desarrollo del mercado interno a una orientada a la apertura comercial, circunstancia que ha propiciado la incursión a un esquema de competitividad productiva a escala global. El tercero se encuentra en la desregulación de los mercados de trabajo, cuyo impacto se expresa en nuevas formas de contratación, aprovechamiento, capacitación y movilidad de la mano de obra en diferentes esferas productivas. El cuarto factor está vinculado con los procesos de reestructuración productiva y los cambios en la organización del trabajo que suponen adecuaciones en las condiciones sociotécnicas de las empresas. Finalmente, el quinto se relaciona con el avance de las tecnologías de la información y comunicación (TIC), cuyos usos se extienden a diversos campos de la actividad humana.

En este marco, la incorporación del término "gestión" en la jerga educativa ha venido progresivamente a sustituir la noción de "administración escolar". Dicho cambio no solo opera en el terreno

1 Esto último no sugiere que dentro del ámbito de la educación se haya descubierto "de repente" el término gestión, como si fuese un concepto de nuevo cuño, siendo que ha estado presente desde hace mucho tiempo en la ciencia política y en las teorías de la organización, particularmente. Su singular uso en esta esfera responde a una resignificación de lo semántico, en la práctica supone introducir una reestructuración del funcionamiento de los centros basada en una racionalidad organizacional emergente fincada en propósitos de política educativa de validez global (Del Castillo y Azuma, 2009; Namo de Mello y Da Silva, 2004). Sobre esta lógica, la gestión escolar se asocia fuertemente con la idea de cambio educativo por cuanto implica la instrumentación de un marco de acción alejado del paradigma burocrático caracterizado por la verticalidad de las decisiones, la estricta división del trabajo, la estandarización de los roles, la marcada separación entre lo administrativo y lo pedagógico, así como el descuido del aprendizaje como eje articulador de la educación. Bajo esta redefinición han incursionado diversos tratamientos y líneas de interés que buscan situar el problema de la gestión dentro de un marco de posibilidades para cubrir las expectativas educativas de nuestro tiempo. De ahí la necesidad de hacer un balance encaminado a la revisión de algunos elementos relevantes que estructuran, de manera general, el estado que guarda el análisis sobre este importante tópico en los países de América Latina².

\section{La gestión escolar como lógica de acción emergente}

Dentro de un primer bloque de autores, el estudio de la gestión se centra en perfilar una serie de dispositivos organizacionales para la mejora de los establecimientos escolares. La principal preocupación está enfocada en destacar los límites del modelo burocrático y su contraste con situaciones

de las formas de organización escolar en el marco de las reformas educativas implementadas desde la década de los ochenta. Ello como parte de la necesidad de instaurar mecanismos de autogestión en línea directa con la descentralización del servicio público, y que definiera el sentido de la reforma del Estado en los países latinoamericanos en los primeros años del neoliberalismo.

2 El presente ejercicio no supone una revisión exhaustiva, amplia y definitiva sobre el tema dentro del panorama latinoamericano; más bien persigue modestamente delinear algunos puntos que definen, en lo general, las vertientes de análisis seguidas por distintos autores preocupados por la gestión en la esfera educativa. 
deseables en línea directa con la descentralización y la autonomía escolar. En esta perspectiva, se presenta un esfuerzo por ubicar la gestión más allá del tamiz administrativo con el que frecuentemente se le asocia, e insertarla como una acción global encaminada a garantizar los propósitos educativos en el interior de las escuelas. Así, por ejemplo, Pilar Pozner advierte que la gestión debe ser el instrumento para recuperar el sentido y la significación de las prácticas pedagógicas a través de una participación más activa de los distintos agentes implicados. Esto supone incluir procesos de autogestión a través de los "proyectos escolares"; un mecanismo por medio del cual se convoca a la comunidad educativa para asegurar la mejora de los aprendizajes en función de las condiciones y necesidades de los centros. Desde este ángulo, la escuela se erige como la unidad educativa fundamental, en tanto la gestión se define como "el conjunto de acciones relacionadas entre sí emprendidas por el equipo directivo [...] para promover y posibilitar la consecución de la intencionalidad pedagógica en-con la comunidad educativa" (Pozner, 2003, p. 35).

En el mismo sentido se pronuncia Elizondo (2005), para quien la gestión escolar implica la auto-organización de la escuela como una forma de favorecer una autonomía y una identidad que le permita decidir sus quehaceres y llevar a cabo los ajustes pertinentes para enfrentar distintas realidades. De este modo, los centros deben pensarse como "organizaciones flexibles"; es decir, permeables a los cambios del entorno, funcionalmente adaptables al carácter contingente de la educación y capaces de brindar resolución a cualquier problema educativo en dondequiera que este se presente. Para Inés Aguerrondo $(1996,2008)$ esta misma posibilidad exige, por un lado, dotar a los establecimientos escolares de la autonomía suficiente para reconocer y corregir diversos procesos en su interior; y por el otro, lograr constituir equipos de trabajo docente en los que sea posible compartir saberes complementarios. Esta condición conduciría a la conformación de una "organización inteligente"; esto es, un escenario escolar abierto al aprendizaje, con capacidad de transformación permanente y con un compromiso constante por la mejora educativa entre sus diferentes miembros. Para conseguir tales fines, la autora propone una serie de acciones: i) disminución de las jerarquías a través de la constitución de equipos autónomos; ii) creación de espacios de intercambio y reflexión continua; iii) incremento del tiempo efectivo del cuerpo docente en las aulas; iv) reconfiguración de la escuela para atender de manera óptima las necesidades de los alumnos; y v) realización de mayores innovaciones para adecuarse a las demandas cambiantes del ambiente (1996, p. 13).

Dentro de esta misma vertiente hay otro grupo de autores (Cervantes, 1998; Graffe, 2002; SchmeIkes, 1994, 2002; Uribe, 2007) cuyo interés descansa en establecer las posibles correspondencias entre la gestión escolar, la calidad educativa y la acción directiva. El elemento común es considerar que la preocupación sustantiva de los establecimientos escolares debe estar enfocada a mejorar los aprendizajes de los alumnos, para lo cual se requiere un responsable (por lo general el directivo) que asuma el quehacer central de la escuela y edifique un liderazgo a fin de generar voluntades orientadas a maximizar el potencial de los estudiantes en el ámbito de su formación. Este esfuerzo por parte del cuerpo directivo tiene que articularse, tanto con el trabajo colegiado de los docentes como con la intervención de la comunidad educativa en su conjunto. La clave de la mejora, por tanto, no recae en un solo aspecto dentro del entramado educativo, sino en la interrelación de distintos procesos emprendidos por los participantes bajo una sólida conducción directiva.

Como puede apreciarse, la línea de estos trabajos está marcada por un fuerte matiz prescriptivo e ilustrativo en torno al funcionamiento de las escuelas como parte de los procesos de descentralización. La base de la argumentación es presentar un esquema de gestión escolar en correspondencia directa con las orientaciones de la nueva gerencia (new ma- 
nagement) pensadas para el ámbito educativo. Así, el análisis de la gestión se desenvuelve, en esencia, en el terreno de lo idóneo o de las propuestas ad hoc con los procesos de reforma educativa iniciados desde la década de los ochenta. No obstante, la principal limitante de esta perspectiva reside en ubicar la mejora de las escuelas como una constante organizacional asociada a la superación indubitable del ordenamiento burocrático, sin discutir en profundidad los diversos grados de racionalidad y burocratización construidos históricamente en múltiples contextos escolares.

\section{La gestión escolar en el marco de la descentralización}

En un segundo eje de aproximación, el interés está centrado en discutir los alcances y los contenidos prácticos de los procesos de descentralización en el contexto de las reformas educativas. En esta vertiente ya no se trata de justificar la implementación de un nuevo modelo de gestión per se, sino poner de relieve las convergencias y los contrastes derivados de la puesta en marcha de mecanismos de autogestión sobre la base de evidencias empíricas. Para fines de ordenación, este conjunto de trabajos se divide en dos momentos o periodos concretos: el primero destaca algunas experiencias de autonomía escolar durante la década de los noventa, mientras el segundo resalta las implicaciones de la gestión en los procesos de evaluación educativa del año 2000 a la fecha.

En un primer momento, los estudios empíricos analizan los efectos de la descentralización en realidades educativas variadas y complejas. En el contexto brasileño, Krawczyk (1999) advierte que las propuestas encaminadas a la autonomía escolar no siguen un patrón único o absoluto; más bien parten de prioridades múltiples y contrapuestas. Esto es así debido a la diversidad de preferencias políticas de los municipios que actúan como mediadores entre la política educativa y su concreción efectiva en las escuelas. En consecuencia, existen modelos variados de gestión no solo por la heterogeneidad de los contextos regionales dentro del Brasil, sino además por los diversos grados de articulación política entre los gobiernos municipales y los establecimientos educativos. De ahí que las concepciones sobre la mejora escolar entre estos dos dominios suelan partir de puntos diferentes, lo cual suscita la aplicación parcial de aspectos clave como la administración adecuada de recursos, la organización de tareas en función de los proyectos escolares o la selección de procedimientos adecuados para lograr los resultados esperados.

En ese mismo contexto, Marília Fonseca (2003) encuentra que si bien la descentralización ha logrado una planeación más organizada y participativa en el interior de los centros, la metodología de trabajo extiende los mecanismos de regulación, control y validación de las tareas. Con ello se contribuye a una mayor burocratización del ejercicio escolar, situación que para muchos docentes solo representa una continuidad de prácticas ya vividas. Contrario a la urgencia de adoptar esquemas de participación flexibles condensados en el Plan de Desempeño Escolar (PDE), instrumento financiado por el Banco Mundial e introducido en escuelas de educación básica, la autora descubre que en realidad se imprime una organización del trabajo muy próxima a la racionalidad taylorista3; es decir, fragmentación de las acciones escolares, división pormenorizada del trabajo y una separación notable entre quienes deciden y quienes ejecutan. A partir de esto, Fonseca apunta la presencia de una "simulación modernizante" en las escuelas estudiadas que no rompe con

3 Frederick Taylor (1856-1915) inauguró una vertiente administrativa fundada en un prototipo de racionalidad mecanicista como forma de garantizar la eficiencia productiva. Esto supuso la incorporación de mecanismos organizacionales basados en la separación estricta entre planeación y ejecución, la verticalidad de las decisiones así como la fragmentación y estandarización de las tareas, entre otros rasgos definitorios de lo que él mismo denominó "administración científica". Este modelo de racionalización, pensado originalmente para la organización industrial y el cual tuvo un impacto importante en buena parte del siglo XX, se trasladó a otras esferas, incluidas desde luego las escuelas, bajo la promesa de ser la única vía por la cual se alcanzaría la modernización institucional de las sociedades capitalistas. 
las verdaderas causas del fracaso escolar, ni tampoco aborda con plenitud las cuestiones pedagógicas.

En la forma como los procesos de reforma son adoptados y recibidos intervienen no solo factores de carácter contextual o institucional, sino también significados diversos alrededor de un principio o una norma de acción. En tal perspectiva, el estudio cualitativo llevado a cabo por Fuller y Rivarola (1998) en doce escuelas de nivel básico en Nicaragua revela la variedad de interpretaciones que tiene para los actores educativos la idea de autonomía escolar. Para estos autores, la iniciativa de reforma establecida desde 1993 en el país centroamericano apela por un nuevo vocabulario sobre cómo motivar la acción escolar; solo que en su decodificación cada agente asume el trabajo con una visión parcialmente informada. Desde ese ángulo, directores y maestros comparten, en general, los contenidos discursivos impresos en la política educativa relativos a la autonomía escolar; sin embargo, los énfasis en cuanto a sus implicaciones prácticas presentan variaciones importantes ya que mientras algunos remarcan la necesidad de independencia con respecto al $\mathrm{Mi}$ nisterio de Educación, sobre todo en lo referente al financiamiento, otros acentúan el aspecto profesionalizante así como la implicación de los padres de familia en los procesos de enseñanza-aprendizaje. Estos contrastes no solo responden a las preferencias de cada actor educativo, sino a la variabilidad de las condiciones en que actúan las escuelas.

En un segundo momento, la atención se centra en destacar las implicancias de la gestión escolar en los resultados de las pruebas estandarizadas. Ante las exigencias institucionales por alcanzar niveles de calidad comparables en el terreno del aprendizaje, se vuelve ineludible la referencia a los factores organizacionales como elementos que marcan diferencias importantes entre los diversos establecimientos. Para Pablo López (2010), esta circunstancia supone indagar la relación proceso-producto en las escuelas para descubrir sus alcances efectivos en el marco de las evaluaciones tanto nacionales como internacionales. De esta forma, el autor considera que para el caso chileno las pruebas estandarizadas han arrojado resultados poco satisfactorios en razón de tres factores clave: 1) ausencia de un liderazgo efectivo entre quienes dirigen; 2) desconocimiento de las herramientas modernas de gestión; y 3) existencia de una cultura organizacional arraigada y opuesta a los cambios. De ahí la necesidad de promover institucionalmente un modelo orientado a la articulación entre el liderazgo, la planificación, los recursos y los resultados. Del mismo modo, Murillo y Román (2009) en un estudio comparativo entre 17 países de América Latina y el Caribe para evaluar los desempeños educativos en alumnos de primaria, encuentran que la variable organizacional posee un peso significativo en los niveles de aprovechamiento. Así, las escuelas en donde los directores cuentan con mayor experiencia en los cargos junto con una formación inicial o permanente relacionada con la gestión, o en donde los profesores trabajan colaborativamente y tienen mayores expectativas hacia sus alumnos, son las que presentan los mejores resultados.

De esta revisión se destaca el hecho de entender que entre las propuestas educativas y las prácticas escolares median elementos contextuales y subjetivos, los cuales influyen en los modos de actuación concretos dentro de los centros. En este caso, los mecanismos de autogestión son recibidos e interpretados de diversas formas, por lo que sus posibilidades reales en cuanto a favorecer procesos de mejora no siguen una ruta de un solo sentido; más bien se entretejen dentro de lógicas institucionales que permiten aperturas o restricciones (parciales o totales) alrededor del cambio educativo. Del mismo modo, las realidades escolares no se pueden concebir como simples escenarios de "ajuste automático" a las exigencias coyunturales, debido a que en su interior confluyen prácticas y experiencias con fuertes arraigos que afectan el carácter de su organización a lo largo del tiempo. Esta circunstancia debe entonces motivar la necesidad de analizar el funciona- 
miento de las escuelas en términos de proceso, y no únicamente a partir de si las prácticas corresponden o no a una disposición oficial en específico.

\section{La gestión desde su dimensión micropolítica}

En un tercer ángulo de aproximación, la gestión se analiza a partir de su carácter relacional así como de la divergencia de razonamientos que dan pie a la configuración de oposiciones, negociaciones o conflictos alrededor de una intencionalidad educativa. Siguiendo una vertiente de reflexión centrada en el asunto del poder para explicar el comportamiento organizacional, la cual se puede ubicar con Crozier y Friedberg (1990) y específicamente con Ball (1989, 2001) para la esfera escolar, este cuerpo de trabajos se aboca a identificar la condición micropolítica presente en los centros. Desde este referente, autores como Alfredo Furlán et al. (2004) establecen que alrededor de la gestión irrumpen variados procesos de decisión entre los agentes del plantel así como marcadas asimetrías en los discursos de sus protagonistas. Sobre esa base, el poder de decisión adquiere un carácter multiforme al tiempo de descansar en distintos puntos de anclaje dentro de la institución; de ello deriva el imperativo de atender el problema del poder y la autoridad para dar cuenta de cómo se estructuran los sistemas escolares en función de la puesta en marcha de los programas pedagógicos.

Por otra parte, Graciela Batallán (2003) advierte que la dinámica escolar se encuentra atravesada por distintas manifestaciones de poder derivadas de los mandatos de las políticas educativas y su articulación con el ejercicio docente. Para la autora, la problemática del poder se envuelve en la sedimentación de las tradiciones doctrinarias, las características estructurales de la actividad escolar, los procesos políticos que conforman el tipo de Estado y las diferentes estrategias que se ponen en juego por los participantes frente a las políticas contingentes. Al amparo de esta compleja trama, los docentes, en particular, encuentran serias contradicciones en las exigencias de cambio en razón de promoverse estas últimas en espacios muy burocráticos y centralizados. Esto hace que en vez de ser reconocidos como responsables de una tarea intelectual se conviertan en la práctica en simples ejecutores de un saber producido por otros. En la misma línea de interés, Jiménez y Perales (2007), a partir de un estudio de caso en Coahuila (México), identifican la gestión como una expresión de verticalidad en las decisiones acompañada de una fragmentación de las responsabilidades y el confinamiento del poder. El sentido de la participación viene marcado así por una "definición de la territorialidad"; es decir, por una demarcación de los ámbitos de poder de cada uno de los miembros de la organización, lo cual conduce a la configuración de núcleos de identidad ya sea para responder a la tradición, o bien para adecuarse a la emergencia normativa desde particulares estrategias de acción. De este modo, la gestión se caracteriza por una distribución de privilegios o una repartición de culpas hecha por la dirección como forma de mantener el control entre los integrantes. Al concentrar la esfera directiva las decisiones importantes se genera una personalización del poder a través de una visión patrimonialista y caciquil, con ello se coloniza la razón de ser de la escuela como parte de una herencia cultural.

Desde una reflexión centrada en analizar los alcances del empoderamiento como elemento estratégico de la nueva gestión, González (2001) plantea que los planes, programas y proyectos de cambio organizacional utilizados en el sistema educativo costarricense abundan en contradicciones internas, lo cual más que estimular la motivación y el compromiso docente lo frena sensiblemente. En términos declarativos a los directivos les parece pertinente delegar el poder entre el personal; sin embargo, el modelo en el que más confían es el del mando y el control directo. La repercusión de esto para el personal docente es entender su quehacer como parte de un compromiso externo, esto es, asumir su labor en fun- 
ción de un deber contractual establecido a priori que les deja poco margen de control sobre sus funciones.

En el mismo orden, Justa Ezpeleta (2004a) considera que las propuestas de innovación no son respuestas incontrovertibles a los rezagos presentes en materia educativa; antes bien, pueden ser motivo de rechazo por quienes tienen la responsabilidad de implementarlas. En tal virtud, las innovaciones no son inseparables de los contextos y los procesos institucionales, por lo que adquieren un sentido político y no solamente técnico-instrumental. Para esta autora, el discurso de la innovación descansa en la presunción de revertir el confinamiento de la enseñanza al ámbito exclusivo del aula para situarla como prioridad en la conducción y finalidad de cada plantel. Pero para ello no basta adherirse a los contenidos de un plan, es necesario vincular esta necesidad de cambio con el diseño histórico de la institución, las definiciones de poder escolar y los compromisos corporativos entre la autoridad educativa y el sindicato magisterial.

La relevancia de estos aportes reside en situar las relaciones de poder como un aspecto fundamental para comprender el carácter de la acción social en el interior de los centros de enseñanza. La recuperación de la dimensión micropolítica nos ofrece la posibilidad de analizar cómo alrededor de un principio educativo se generan posicionamientos -latentes o manifiestos - entre los integrantes de la organización, a partir de lo cual se determina el alcance efectivo de las implementaciones normativas. Esta consideración permite además reconocer el papel que desempeñan los razonamientos diversos, las alianzas, los intereses puestos en juego, las ideologías acerca de lo pedagógico así como las relaciones de fuerza presentes entre los participantes para definir y sostener una práctica educativa. La gestión, en consecuencia, no puede analizarse como una acción transparente, funcionalmente adaptable a las circunstancias y libre de revisiones o controversias; antes bien, como un componente en el que se expresan situaciones sociales en donde las motivaciones de los sujetos suelen ser ambiguas, inciertas y contradictorias.

\section{Los marcos conceptuales de la gestión escolar}

Una última vertiente de estudio la podemos situar en aquellos tratamientos preocupados por establecer demarcaciones analíticas y conceptuales alrededor de la gestión en las escuelas. A la par de las redefiniciones de las políticas educativas desde la década de 1980 en América Latina, se han desarrollado reflexiones orientadas a otorgarle a la gestión escolar un canon disciplinar propio a partir de la recuperación de elementos asociados a las teorías de la organización, así como de contenidos relacionados directamente con el área educativa. Con sus respectivos matices, estas perspectivas se concentran, en lo fundamental, en torno al problema de la definición del objeto de la gestión en los procesos educativos y sus implicaciones metodológicas.

En este esfuerzo de demarcación existen posturas que proponen la necesidad de conceptualizar la gestión escolar más allá de una visión meramente gerencial o circunscrita al horizonte organizacional, sin establecer claramente sus lógicas de articulación con las políticas públicas así como con los dispositivos institucionales que le dan sentido al sistema educativo. A ese nivel, autores como Juan Cassasus (2000) y Lucía Rivera (2010) enfatizan la presencia de múltiples procesos en torno a la gestión que abarcan una gran diversidad de actores, recursos y actividades en diferentes órdenes. Este acercamiento supone concebir la gestión inserta en un entramado a través del cual convergen elementos de carácter institucional, organizacional, político y pragmático que inciden en la definición de las prácticas educativas en el interior de los centros. Para Cassasus, esta condición exige explicar la naturaleza de la gestión, por un lado, como parte de una narrativa condensada en las políticas educativas, cuyo dominio prescriptivo presiona "desde arriba" para hacer ajustes en la práctica con- 
creta; y, por otro, como un principio de organización del trabajo vinculado con el ámbito de la educación, tanto en lo disciplinar como en lo político. Por su parte, Rivera considera oportuno atraer metodológicamente la dimensión institucional-organizacional como una herramienta conceptual para analizar la relación dialéctica entre las prácticas, la institucionalización de los conocimientos y los procesos organizacionales de las escuelas sobre los cuales se materializan las concepciones, valores, saberes y representaciones del mundo social.

Dentro de esta misma vertiente, existe otra posición orientada a repensar la gestión escolar desde su propia especificidad; esto es, a partir de los elementos constitutivos que la hacen distinta a la localizada en otras organizaciones, especialmente las empresariales. A ese respecto, Susana Germán (2003) advierte en las escuelas escenarios que presentan un alto grado de diversificación expresado en diferentes modalidades de enseñanza y bajo la intervención de ciertos actores quienes, en principio, resultan ajenos a la organización, como los padres de familia o los vecinos, lo cual habilita la posibilidad de desarrollar procesos en otros ámbitos como el barrio, las plazas públicas, las unidades familiares, los centros de recreación, etc. Bajo este fundamento, la autora centra el análisis de la gestión desde la interacción comunicativa y ubica su especificidad en el predominio de las relaciones interactivas e intersubjetivas; en la fuerte incidencia de los componentes emocionales, valorativos e ideológicos vinculados con la finalidad pedagógica; y en la presencia de funciones que, en determinadas condiciones, desbordan el cumplimiento educativo, tales como acciones asistenciales, sociocomunitarias o económico-productivas, entre otros aspectos.

Del mismo modo, Ezpeleta (2004b) conviene en la necesidad de aproximarse a la escuela a partir de su cotidianidad imbricada en particulares procesos de acción social. Sobre este principio teórico trata de situar la gestión como una práctica cotidiana en la cual se estructuran modos de existen- cia escolar no condicionados necesariamente por lo normativo, sino a través de la coexistencia de tres ordenamientos: 1) el técnico-pedagógico, 2) el administrativo y 3) el laboral. La interrelación de estos componentes configura una "modalidad institucional"; una categoría que describe "la confluencia y combinación de características significativas determinadas por la administración y la organización escolares" (pp. 112, 113).

De estos aportes se recupera la pertinencia de trazar la gestión desde diferentes niveles de articulación que la afectan y la componen. En términos teórico-metodológicos, esto último implica estudiar la escuela como parte de un entramado de relaciones más amplio que desborda sus propios límites, por cuanto su presencia está mediada por elementos de diversa magnitud y complejidad. La apuesta, por tanto, es continuar este sendero abierto con el propósito de aproximarnos a las realidades escolares desde distintos ángulos como una vía para trascender las concepciones funcionalistas centradas esencialmente en lo normativo, las cuales dominan en la actualidad los proyectos político-pedagógicos. Sin embargo, es necesario también incluir la discusión de la gestión escolar desde fundamentos más epistémicos; es decir, a través de la incorporación de la acción social y la relación sujeto-objeto como referentes de análisis que deriven en una mirada multidisciplinar a fin de ampliar el horizonte de acercamiento teórico-metodológico.

\section{Un apunte final}

Por lo anteriormente expuesto, consideramos que alrededor de la gestión en los centros de enseñanza básica hay todavía mucho por discutir en razón de constituirse en una veta abierta para proseguir en su reflexión, conceptualización y tratamiento empírico. A lo largo de estas tres últimas décadas, los procesos de reforma educativa en Latinoamérica han buscado dar un reordenamiento a diferentes componentes que configuran el entrama- 
do escolar. En este punto, la gestión se ha convertido en un asunto de interés particular por cuanto, por una parte, concentra los ideales de funcionamiento organizacional que deben seguir los centros educativos de cara a las exigencias de una sociedad altamente cambiante y diversificada; y por otra, refleja en su concreción efectiva la variedad de intereses, expectativas, motivaciones y posicionamientos que los distintos agentes desarrollan en sus relaciones mutuas sobre la base de determinados propósitos educativos. La relevancia de su estudio se acrecienta a medida que en la escuela se depositan nuevas expectativas de formación acordes con las transformaciones que se están produciendo globalmente, lo cual marca un escenario de constante redefinición de los fines educativos con implicaciones directas en las formas de actuación en el interior de los centros de enseñanza.

\section{Referencias}

Aguerrondo, I. (1996). La escuela como organización inteligente. Argentina: Troquel.

Aguerrondo, I. (2008). La influencia del contexto en la efectividad de la escuela. Consideraciones para el desarrollo profesional docente. En Unesco/Llece, Eficacia escolar y factores asociados en América Latina y el Caribe. Santiago de Chile: Unesco.

Ball, S. (1989). La micropolitica en la escuela. Hacia una teoría de la organización escolar. Madrid: Paidós Ibérica.

Ball, S. (2001). La gestión como tecnología moral. En Ball, S. (comp.). Foucault y la educación. Disciplinas y saber. Madrid: Morata.

Batallán, G. (2003). El poder y la autoridad en la escuela. La conflictividad de las relaciones escolares desde la perspectiva de los docentes de infancia. Revista Mexicana de Investigación Educativa (RMIE), 8 (19), 679-704.

Cassasus, J. (2000). Problemas de la gestión educativa en América Latina (La tensión de los paradigmas de Tipo A y el Tipo B). (Versión preliminar). Unesco, pp. 1-25.

Cervantes Galván, E. (1998). Una cultura de calidad en la escuela, liderazgo para el cambio educativo. México: Ediciones Castillo.

Crozier, M. y Friedberg, E. (1990). El actor y el sistema: las restricciones de la acción colectiva. México: FCE.

Del Castillo, G. y Azuma, A. (2009). La reforma y las politicas educativas. Impacto en la supervisión escolar. México: Flacso.

Elizondo, A. (coord.) (2005). La nueva escuela. Dirección, liderazgo y gestión escolar (t. I). México: Paidós.

Ezpeleta, J. (2004a). Innovaciones educativas. Reflexiones sobre los contextos en su implementación. Revista Mexicana de Investigación Educativa, 9 (21), 403-424. 
ISSN $0123-1294$ | Educ.Educ. Vol. 17. No. 2 | Mayo-Agosto de 2014 | pp. 357-369.

Universidad de La Sabana | Facultad de Educación

Ezpeleta, J. (2004b). Problemas y teoría a propósito de la gestión pedagógica. En Ezpeleta, J. y Furlán, A. (comps.). La gestión pedagógica de la escuela (pp. 101-117). México: Unesco.

Fonseca, M. (2003). O projeto político-pedagógico e o plano de desenvolvimiento da escola: duas concepções antagónicas de gestão escolar. Cad. Cedes Campinas, 23 (61), 302-318.

Fuller, B. \& Rivarola, M. (1998). Nicaragua's experiment to decentralize schools: view of parents, teachers and directors. Nicaragua: Ministerio de Educación - Banco Mundial.

Furlán, A. et al. (2004). La gestión pedagógica. Polémicas y casos. En Ezpeleta, J. y Furlán, A. (comps.). La gestión pedagógica de la escuela (pp.138-174). México: Unesco.

Germán Cantero, S. (1999). Un análisis alternativo. En Secretaría de Educación Pública (SEP), Antología de gestión escolar (pp. 61-69). México: SEP.

González Salazar, L. E. (2001). Empoderar: nuevo concepto de liderazgo para el administrador educativo. Revista Educación, 25 (2), 41-47.

Graffe, G. J. (2002). Gestión educativa para la transformación de la escuela. Revista de Pedagogía, 23 (68). Recuperado el 2 de abril de 2013 de: http://www.scielo.org.ve/scielo.php?script=sci_arttext\&pid=So798$97922002000300007 \& \operatorname{lng}=$ es\&nrm=iso.

Jiménez Lozano, M. L. y Perales, F. J. (2007). Entre proyectos personales y propuestas operativas. Etnografía de la gestión en la escuela. Revista Mexicana de Investigación Educativa (RMIE), 12 (35), 1309-1328.

Krawczyk, N. (1999). A gestão escolar: um campo minado... Análise das propostas de 11 municípios brasileiros. Educação \& Sociedade, XX (67), 112-149.

López, P. (2010). Variables asociadas a la gestión escolar como factores de calidad educativa. Estudios Pedagógi$\cos$ XXXVI (1), 147-158.

Murillo Torrecilla, J. (2003). Una panorámica de la investigación iberoamericana sobre eficacia escolar. Revista Electrónica Iberoamericana sobre Calidad, Eficacia y Cambio en Educación (REICE), 1 (OO1), 1-14.

Murillo Torrecilla, J. y Román, M. (2009). Mejorar el desempeño de los estudiantes de América Latina. Revista Mexicana de Investigación Educativa (RMIE), 14 (41), 451-484.

Namo de Mello, G. y Da Silva, T. R. (2004). La gestión en la escuela en las nuevas perspectivas de las políticas educativas. En Ezpeleta, J. y Furlán, A. (comps.). La gestión pedagógica de la escuela (pp. 44-63). México: Unesco.

Pozner de Weinberg, P. (2003). La gestión escolar. En Secretaría de Educación Pública (SEP). Antología de gestión escolar (pp. 35-58). México: SEP. 
Rivera Ferreiro, L. (2010). Elementos conceptuales para el análisis de las prácticas de gestión educativa. En Guerra, M. (coord.). Gestión de la Educación Básica. Referentes, reflexiones y experiencias de investigación (pp. 71-86). México: SEP-UPN.

Schmelkes, S. (1994). Hacia una mejor calidad de nuestras escuelas. OEA, Colección Interamer, 32. Recuperado el 2 de abril de 2013 de: http://http://www.ctascon.com/Hacia\%2Ouna\%2omejor\%2ocalidad\%20de\%20 las\%2oEscuelas.pdf.

Schmelkes, S. (2002). Calidad de la educación y gestión escolar. En Secretaría de Educación Pública (SEP). Primer curso nacional para directivos de educación primaria. Lecturas (pp. 125-134). México: SEP.

Uribe, M. (2007). Liderazgo y competencias directivas para el liderazgo escolar: experiencia del Modelo de Gestión Escolar de Fundación Chile. Revista Electrónica Iberoamericana sobre Calidad, Eficacia y Cambio en Educación (REICE), 5 (5e), 149-156. 
